\title{
TRIAJE COMO HERRAMIENTA EN EMERGENCIAS PEDIÁTRICAS DEL HOSPITAL INFANTIL DR. ROBERT REID CABRAL SEPTIEMBRE 2018 - NOVIEMBRE 2018
}

\section{Triage as a tool in pediatric emergencies at Dr. Robert Reid Cabral Children's Hospital september 2018 - november 2018}

\section{Anyeliza Quiñones ${ }^{a}$, Cristina Contreras ${ }^{b}$, Lisandra Corona $^{c}$ y Clemente Terrero ${ }^{d}$}

Recibido: 6 de septiembre, 2019 • Aprobado: 2 de diciembre, 2019

Cómo citar: Quiñones A, Contreras C, Corona L, Terrero C. Triaje como herramienta en emergencias pediátricas del Hospital Infantil Dr. Robert Reid Cabral septiembre 2018 - noviembre 2018. cysa [Internet]. 17 de junio de 2020 [citado 17 de junio de 2020];4(2):5-11. Disponible en: https://revistas.intec.edu.do/index.php/cisa/article/view/1768

\section{Resumen}

El triaje busca identificar a aquellos pacientes que necesitan atención inmediata y a los que pueden esperar con seguridad durante un tiempo más prolongado. Se realizó un estudio observacional, descriptivo y prospectivo, con pacientes que acuden al servicio de emergencias del Hospital Infantil Dr. Robert Reid Cabral en los meses de septiembre, octubre y noviembre de 2018, para un total de $32 \%$, $21 \%$, y $47 \%$, respectivamente. El total de pacientes captados fue 660, $54 \%$ masculinos y $46 \%$ femeninos. Edad de mayor asistencia de 1-6 años, 311 para un $47 \%$. El 4to nivel fue el de mayor atención, 219 para un $33 \%$. Mayor flujo horario de 11:00 a.m. a 4:00 p.m., para un $5.8 \%$ y $6.1 \%$. Tiempo de respuesta nivel 1: $50 \mathrm{seg}$; nivel 2: $22 \mathrm{~min}$ con $5 \mathrm{seg}$; nivel 3: $41 \mathrm{~min}$ con $12 \mathrm{seg}$; nivel 4: $49 \mathrm{~min}$ con $35 \mathrm{seg}$; nivel 5: 48 min con $53 \mathrm{seg}$. Como conclusión, la respuesta a las atenciones se ve afectada por la falta de triaje estructurado, una respuesta más rápida y oportuna a pacientes con niveles 3 a 4 , gracias al alto volumen de pacientes que acuden y no así a los de nivel 1 y 2.

Palabras clave: triage; pediatría; emergencia; clasificación; nivel.

\footnotetext{
a Dra. en Pediatría, Titulo en Emergencias Pediátricas, Hospital Infantil Dr. Robert Reid Cabral, República Dominicana.

ORCID: 0000-0001-8202-7108, Correo-e: qanyeliza@gmail.com

b Dra. en Pediatría, Coordinadora de la Escuela de Emergencias Pediátricas, Hospital Infantil Dr. Robert Reid Cabral, República Dominicana.

ORCID: 0000-002-9735-2736, Correo-e: cristina.contreras@intec.edu.do
}

\begin{abstract}
The triage seeks to identify those who need immediate attention and those who can wait safely for a longer time. An observational, descriptive and prospective study was conducted, with patients attending the emergency service of the Dr. Robert Reid Cabral Children's Hospital in the months of September, October and November 2018, for a total of $32 \%, 21 \%$, and $47 \%$ respectively. The total number of patients recruited was 660, $54 \%$ male and $46 \%$ female. Age of greatest attendance of 1-6 years old, 311 for $47 \%$. The 4 th level was the one with the highest attention, 219 for 33\%. Highest hourly flow from 11:00 a.m. to $4: 00$ p.m. with a $5.8 \%$ and $6.1 \%$. Response time level 1: $50 \mathrm{sec}$; level 2: $22 \mathrm{~min}$ with $5 \mathrm{sec}$; level 3: $41 \mathrm{~min}$ with $12 \mathrm{sec}$; level 4: $49 \mathrm{~min}$ with $35 \mathrm{sec}$; and level 5: $48 \mathrm{~min}$ with 53 sec. Conclusion: the response to care is affected by the lack of structured triage, a faster and more timely response to patients with levels 3 to 4 , thanks to the high volume of patients who attend and not to those of level 1 and 2 .
\end{abstract}

Keywords: Triage; pediatrics; emergency classification; Level.

\footnotetext{
${ }^{c}$ Dra. en Pediatría, Gerente General Dpto. Emergencias Pediátricas, Hospital Infantil Dr. Robert Reid Cabral, República Dominicana. ORCID: 0000-0002-7353-7330, Correo-e: lcoronaq@33gmail.com

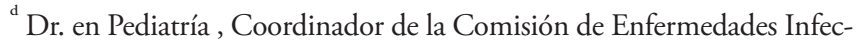
tocontagiosas del Colegio Médico Dominicano. Subdirector, Hospital Infantil Dr. Robert Reid Cabral, República Dominicana.
} 


\section{Introducción}

El triaje es un término que proviene del francés trier y se define como escoger, separar o clasificar. Se emplea en los servicios de emergencia, y resulta necesario debido al aumento de pacientes que acude a este servicio. ${ }^{1}$

En el manual de Advanced Trauma Life Support (ATLS), el triaje se define como el método de selección y clasificación de pacientes, basado en sus necesidades terapéuticas y los recursos disponibles. ${ }^{2}$

Esta definición contempla al triaje como un proceso dinámico, con capacidad de adaptación a factores cambiantes incluso dentro de una misma institución (número de pacientes, hora del día, disponibilidad de recursos, etc.). ${ }^{3}$

Identifica a aquellos pacientes que necesitan atención inmediata y a los que pueden esperar con seguridad durante un tiempo más prolongado, o que quizás no necesiten atención de emergencia. Además, la categoría de urgencia relacionada con el tiempo de espera real se usa como una medida de calidad para los departamentos de emergencia. ${ }^{4}$

El Hospital Infantil Dr. Robert Reid Cabral es el centro pediátrico de referencia nacional de República Dominicana, y cuenta con un sistema de triaje aún deficiente.

En la resolución No. 000022, agosto de 2016, Altagracia Guzmán Marcelino, Ministra de Salud Pública, República Dominicana, ordena que todo establecimiento de salud oferte servicios de atención a emergencias o urgencias aplique un triaje hospitalario. $^{5}$

"Lo urgente no siempre es grave y lo grave no es siempre urgente". 6

Por tal razón, la calidad de atención y del triaje en centros como el Robert Reid Cabral tal vez se vea afectada.
Pese a las condiciones observadas, estudios anteriores reportan que, a través de cuestionarios auto-administrado, para medir la percepción, los resultados más relevantes en cuanto al tiempo de espera en centros como el Hospital Materno-Infantil San Lorenzo de Los Mina fueron un $55 \%$, para muy satisfecho. En total, la percepción que tienen los usuarios que asistieron a la emergencia pediátrica es muy buena con un $84 \%{ }^{7}$

A nivel nacional se reportó que el $40.5 \%$ de los pacientes que acuden a sala de urgencias pertenecen al nivel III o nivel IV de urgencia de la escala de triaje. El $94 \%$ de los pacientes espera 0-2 minutos para ser atendidos y la hora donde más asisten pacientes es entre las 9:00 a. m. y 12:00 p. m. representando el $30 \%$ de los pacientes. El $84.5 \%$ de los pacientes que son evaluados en urgencias son despachados a su domicilio. ${ }^{8}$ Lo cual no aparenta tener cambios hasta la fecha.

\section{Sistemas de triage}

Actualmente existen 5 niveles de triaje: de nivel I a nivel V, de mayor a menor gravedad. Cada nivel va a determinar el tiempo óptimo entre la llegada y la atención médica; así, el tiempo de demora oscila entre la atención inmediata (nivel I) hasta 240 minutos (nivel V).?

$\mathrm{Al}$ igual se reconocen cinco modelos de triaje estructurado con una amplia implantación:

- Australian Triage Scale (ATS)

- Canadian Emergency Department, Triage and Acuity Scale (CTAS)

- Manchester Triage System (MTS)

- Emergency Severity Index (ESI)

- Sistema Español de Triage (SET) adoptado por la Sociedad Española de Medicina de Emergencias (SEMES) a partir del Model Andorrá de Triage: MAT. ${ }^{1}$ 
Hasta la fecha solo la versión pediátrica de la escala de agudeza de triaje canadiense (CTAS), llamada PaedsCTAS, puede representar un indicador alternativo para la vigilancia de la gravedad de pacientes pediátricos. El puntaje PaedCTAS de cinco niveles varía de I (resucitación) a V (no urgente). ${ }^{10}$

\section{Material y método}

Se realizó un estudio de tipo observacional descriptivo, prospective, con el cual se buscó establecer la funcionabilidad del triaje como herramienta en emergencias pediátricas del Hospital Infantil Dr. Robert Reid Cabral en el periodo septiembre 2018
- noviembre 2018. Con pacientes que acudieron al servicio de emergencias del Hospital Infantil Dr. Robert Reid Cabral, de lunes a viernes de 10:00 a. $\mathrm{m}$. a 12:00 p. m.

Los datos fueron recolectados a través de un formulario para pacientes $<1$ año y de 1 a 16 años de edad, que contiene datos socio-demográficos: nombre, edad, sexo, procedencia, número de formulario, fecha, hora de llegada, hora de asistencia, motivo de consulta, comorbilidad, diagrama para identificar el área afectada, triángulo pediátrico y niveles de clasificación.
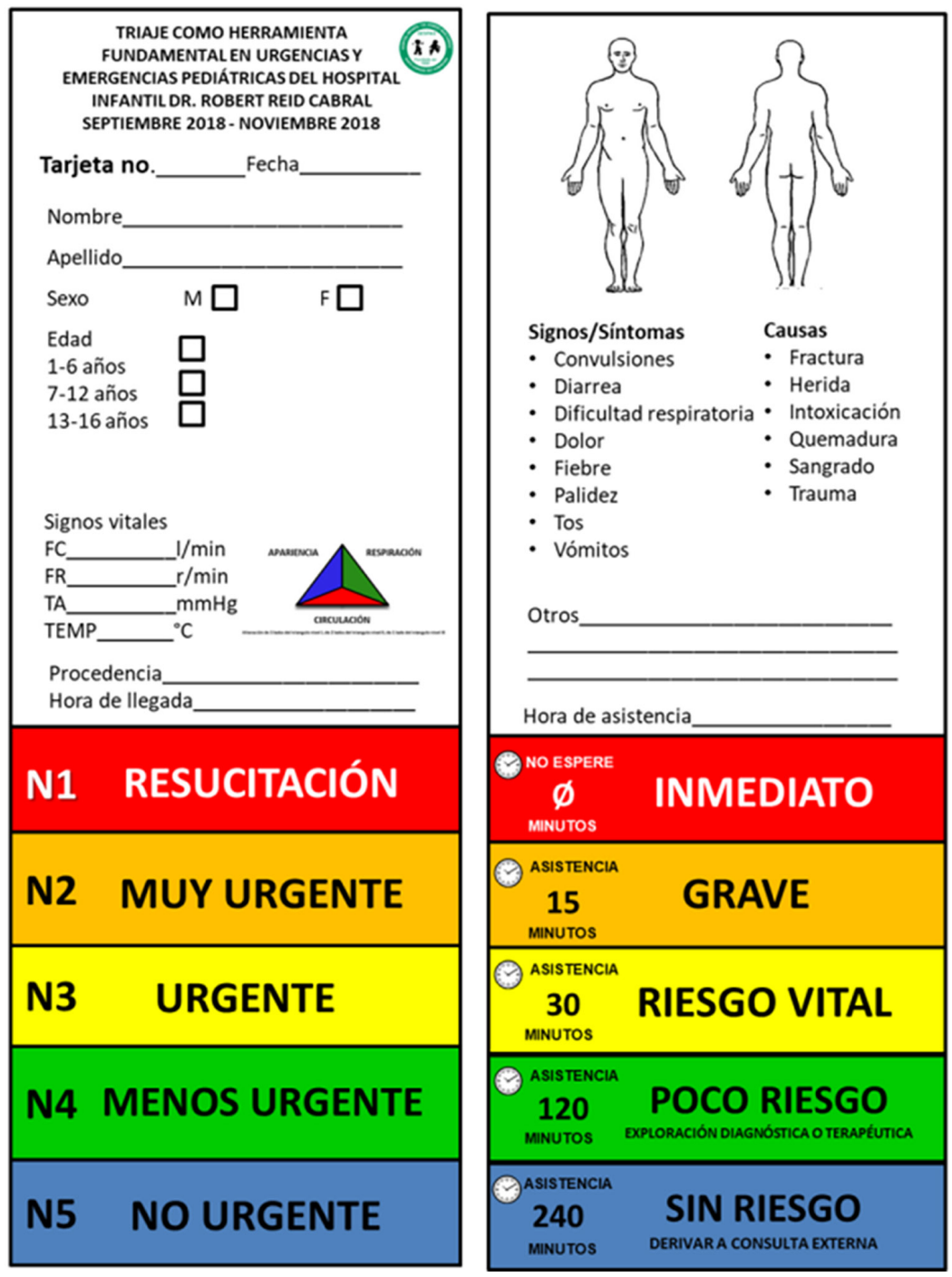

Fuente: archivo Emergencias Hospital Infantil Dr. Robert Reid Cabral. 


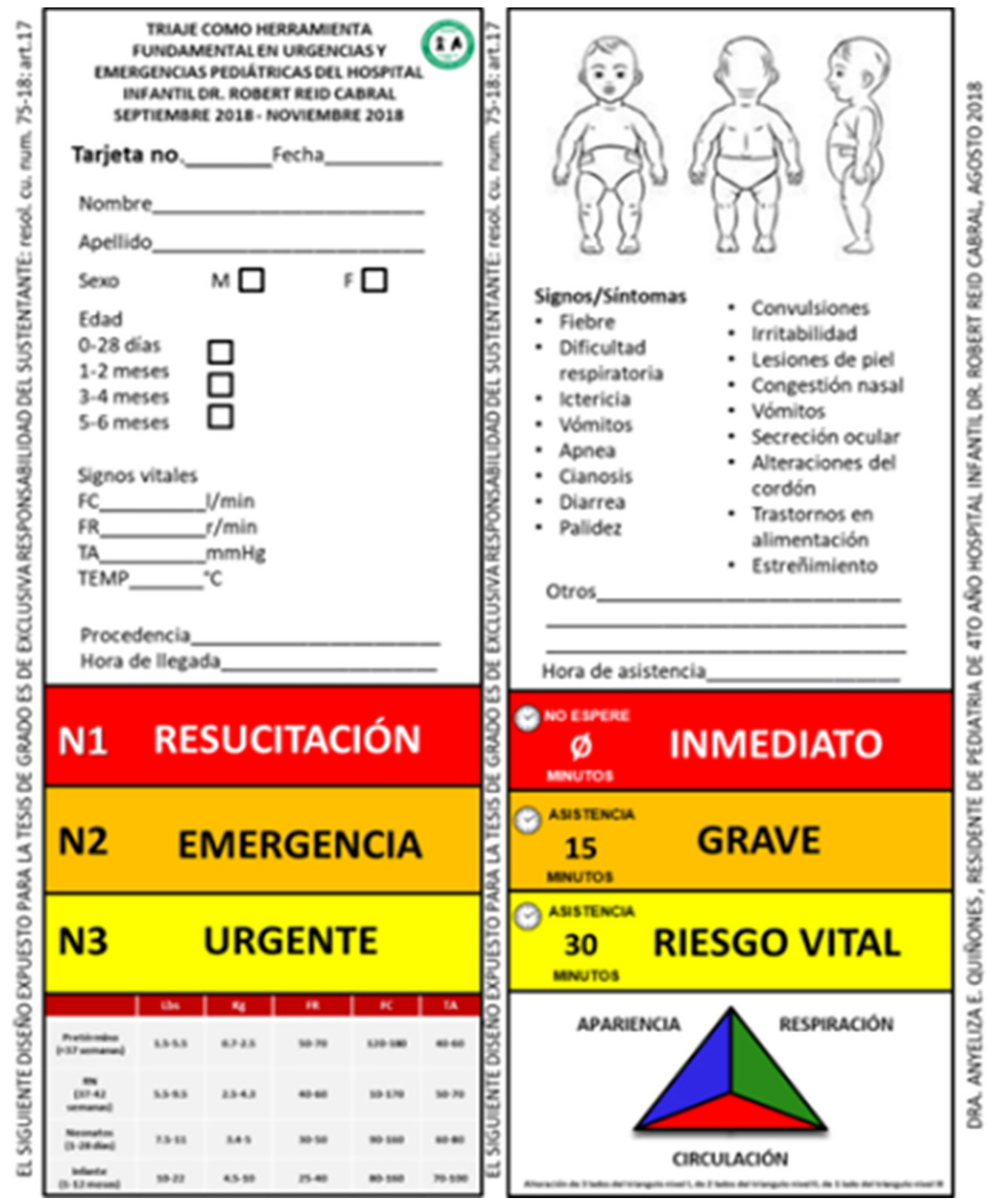

Fuente: archivo Emergencias Hospital Infantil Dr. Robert Reid Cabral.

\section{Resultados}

Se determinó que un $33 \%$ fueron clasificados en nivel 4 un $31 \%$, nivel 3 un $28 \%$, nivel 5 un $7 \%$, nivel 2 un $7 \%$ y $1 \%$ nivel 1 .

La población en estudio procede en mayor porcentaje del Distrito Nacional, con un $71 \%$. El mayor motivo de consulta fue la dificultad respiratoria con un $26 \%$.
En cuanto al flujo de pacientes se determinó que en horarios matutinos de 9:00 a. m. a 11:00 a. m. se presentó un $14.7 \%$ de la población en estudio. En horas vespertinas, de 4:00 p. m a 5:00 p. m. acudieron $15.8 \%$, y en horas nocturnas, desciende considerablemente la asistencia, a partir de las 5:00 p. m. 


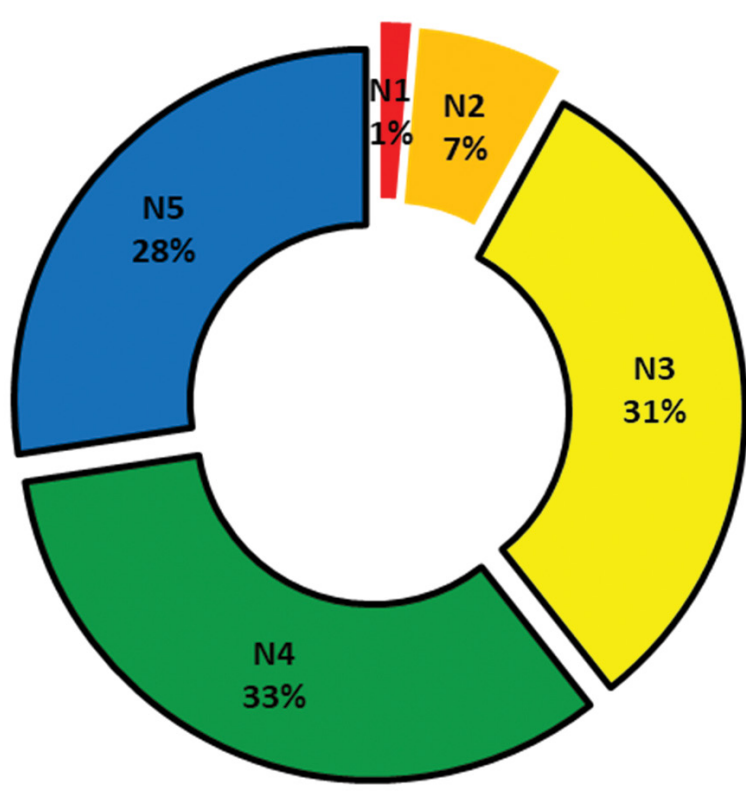

Fuente: archivo Emergencias Hospital Infantil Dr. Robert Reid Cabral.

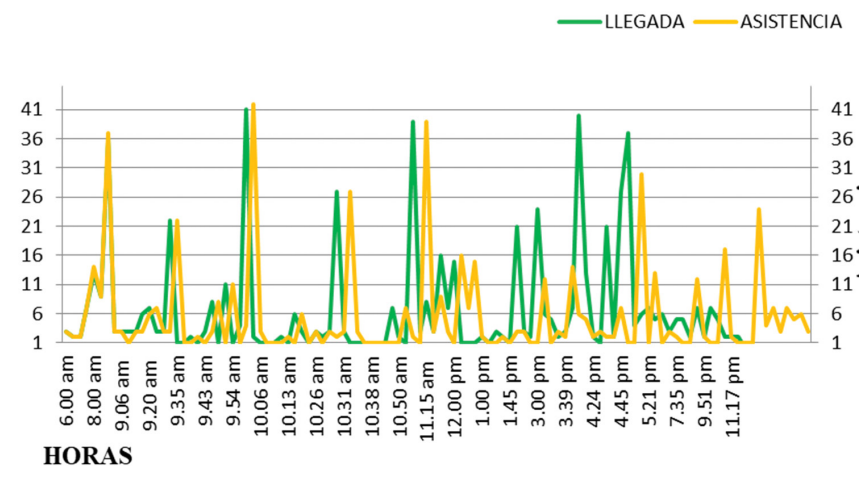

Fuente: archivo Emergencias Hospital Infantil Dr. Robert Reid Cabral.

Se determinó qué tiempo era utilizado por el personal de emergencias una vez realizada la clasificación.

\section{Nivel 1}

\begin{tabular}{|l|c|}
\hline Tiempo total de asistencia & $0: 06: 40$ \\
\hline Tiempo promedio de respuesta & $\mathbf{0 : 0 0 : 5 0}$ \\
\hline Tiempo de respuesta por paciente & $\mathbf{0 : 0 0 : 4 4}$ \\
\hline
\end{tabular}

\section{Nivel 2}

\begin{tabular}{|l|r|}
\hline Tiempo total de asistencia & $8: 28: 00$ \\
\hline Tiempo promedio de respuesta & $\mathbf{0 : 2 2 : 0 5}$ \\
\hline Tiempo de respuesta por paciente & $\mathbf{0 : 1 1 : 3 3}$ \\
\hline
\end{tabular}

\section{Nivel 3}

\begin{tabular}{|l|r|}
\hline Tiempo total de asistencia & $8: 28: 00$ \\
\hline Tiempo promedio de respuesta & $\mathbf{0 : 2 2 : 0 5}$ \\
\hline Tiempo de respuesta por paciente & $\mathbf{0 : 1 1 : 3 3}$ \\
\hline
\end{tabular}

\section{Nivel 4}

\begin{tabular}{|l|r|}
\hline Tiempo total de asistencia & $101: 39: 00$ \\
\hline Tiempo promedio de respuesta & $\mathbf{0 : 4 9 : 3 5}$ \\
\hline Tiempo de respuesta por paciente & $\mathbf{0 : 2 7 : 5 1}$ \\
\hline
\end{tabular}

\section{Nivel 5}

\begin{tabular}{|l|r|}
\hline Tiempo total de asistencia & $79: 50: 00$ \\
\hline Tiempo promedio de respuesta & $\mathbf{0 : 4 8 : 5 3}$ \\
\hline Tiempo de respuesta por paciente & $\mathbf{0 : 2 6 : 2 8}$ \\
\hline
\end{tabular}

Fuente: archivo Emergencias Hospital Infantil Dr. Robert Reid Cabral.

\section{Discusión}

Pese a las deficiencias, estudios de nivel de conocimiento sobre triaje de los residentes de 1er y 3er año de pediatría del Hospital Infantil Dr. Robert Reid Cabral, reportan que de un total de 76 residentes (27 de primer y segundo ańo, y 15 de tercer ańo), solo 3 no tenían conocimiento sobre triaje. El $69.8 \%$ de los residentes considera que debe realizarse en la sala de espera. El $63.2 \%$ considera que el triaje es un proceso que permite la gestión de riesgo cuando la demanda y las necesidades clínicas superan los recursos y, además, que permite una adecuada clasificación del riesgo clínico. En cuanto a la escala utilizada, 23 no han utilizado alguna, 20 han utilizado una de las siguientes: ATS, MTS, ESI, SET y 19 las han utilizado todas. ${ }^{11}$ 
Hasta la fecha, la institución no se ha ajustado a los estándares de tiempo que establecen las guías sobre triaje pediátrico, aun con los conocimientos de su personal presente en emergencias.

Por tal razón, nuestro estudio reportó como tiempos de asistencia para el nivel I: 50 segundos (debe esperar: 0 minutos); nivel II: 22 minutos con 5 segundos (debe esperar: no más de 15 minutos); nivel III: 41 minutos con 5 segundos (debe esperar: 30 minutos); nivel IV: 49 minutos y 35 segundos (debe esperar: no más de 120 minutos); y nivel V: 48 minutos y 53 segundos (debe esperar: no más de 240 minutos).

Lo cual nos demuestra que el tiempo utilizado para la clasificación y atención de pacientes niveles III, $\mathrm{IV}$ y V es en promedio más corto que para los pacientes I y II, los cuales no deben superar 0 y 15 minutos, respectivamente.

Se ha establecido que es necesario poder contar con una estructura funcional del servicio de guardia. ${ }^{12}$

"Todos los sistemas de clasificación pueden verse influidos por una serie de factores que deben ser tenidos en cuenta". ${ }^{13}$

Esto puede ser el resultado del alto volumen de pacientes en sala de emergencias con afecciones menores (niveles III, IV y V) más la necesidad del personal de querer despejar dicho volumen con rapidez.

Todos estos datos de tiempo se relacionan con otros estudios, que dan como resultado que según niveles de gravedad el tiempo de espera para recibir la atención fueron: nivel I: 16,6 minutos; nivel II: 35,41 minutos; nivel III: 42,41 minutos; nivel IV: 45,92 minutos; nivel V: 45,08 minutos. ${ }^{14}$

\section{Conclusión}

La impresión inicial es fundamental, ya que en ocasiones simplemente una visión general del aspecto y de la situación del paciente es suficiente para decidir el nivel de triaje. Esto es más cierto en los niveles más urgentes, mientras que los pacientes a los que se les asigna un nivel IV o $V$ tienen buen aspecto $y$ requieren una sistemática que valore datos no tan evidentes para identificar aquellos factores de riesgo que condicionan el grado de urgencia.

Por tanto, el profesional debe conocer y aplicar el TEP (triángulo de evaluación pediátrica) como actual sistema para realizar una clasificación fisiopatológica. Mientras más lados del triángulo estén alterados, más grave es la situación.

- Si tiene los 3 lados alterados la clasificación es nivel I (rojo)

- Si tiene 2 lados alterados: nivel II (naranja)

- Con un solo lado alterado: nivel III (amarillo)

- Sin ningún lado alterado se aplicarán discriminantes para definir nivel IV o V (edad, afectación aguda o crónica, motivo de consulta). ${ }^{15}$

Podemos finalizar diciendo que los resultados de nuestra investigación fueron beneficiosos ya que arrojan el estado más cercano a la realidad actual de la sala de emergencias del Hospital Infantil Dr. Robert Reid Cabral, en vista de no contar con los recursos tecnológicos para la estratificación exacta de las intervenciones. A pesar de dichas precariedades, los sistemas actualmente utilizados proporcionan un acertado pronóstico a los pacientes triados.

\section{Bibliografía}

1. Soler W, Muñoz MG, Bragulat E, Álvarez A. El Triaje: Herramienta Fundamental en Urgencias y Emergencias. Anales Del Sistema Sanitario De Navarra. 2010;33(suppl.1):55-68

2. Azcona LA, Gutiérrez GE, Fernández CJ, Natera OM, Speare OR-, Ali J. Advanced Trauma Life Support (ATLS) Skills Among 
ATLS Instructors And Providers In México. Journal Of The American College Of Surgeons. 2002;195(3):372-7.

3. Velandia Escobar M. Triage de Urgencia, Revista de Enfermería, Medicina [Internet]. Encolombia.Com. [Cited 26 Sep 2018]. Available From: Https://Encolombia.Com/ Medicina/Revistas-Medicas/Enfermeria/Ve51/Enfermeria5102-Triage/

4. Cruz Pichardom E, Ramos J. Calidad del Triaje en el Servicio de Emergencia del Hospital General Plaza de La Salud. Santo Domingo; 2013. UNIBE

5. Ministerio De Salud Pública, República Dominicana. Resolución No. 000022. Santo Domingo: Año del Fomento de la Vivienda"; 2016. pp. 2-4.

6. Tudela P, Maria Mòdol JM Urgencias Hospitalarias. Medicina Clínica. 2003;120(18):711-6.

7. Medina E, Subervi R, Soto Y. Percepción de la Calidad del Servicio que tienen los Usuarios que asisten a la Emergencia Pediátrica del Hospital Materno-Infantil San Lorenzo De Los Mina. Santo Domingo; 2017.

8. Payano C, Lambertus T. Evaluación de los Servicios de Emergencias Hospitalarias en la Ciudad de Santo Domingo. Santo Domingo; 1963;43(2):50-5.

9. Yates M, Ishikawa T, Schneeberg A, Brussoni M. Pediatric Canadian Triage And Acuity Scale (Paedsctas) As A Measure Of Injury Severity. International Journal Of Environmental Research And Public Health. 2016;13(7):659.
10. Abreu Sánchez P. Nivel de Conocimiento sobre Triaje de los Residentes de 1 y 3 Año de Pediatría del Hospital Infantil Dr. Robert Reid Cabral. Santo Domingo; 2015.

11. Medina J, Ghezzi C, Figueredo D, León D, Rojas G, Cáceres L et al. Triage: Experiencia en un Servicio de Urgencias Pediátricas. Revista Chilena De Pediatría. 2007;78(2):211-6

12. Cialzeta D. Actualización Bibliográfica. Triage: Cómo Categorizar Mejor a los Pacientes en un Departamento de Urgencias Pediátrico. (55th ed.). Buenos Aires, Argentina: Revista Hospital Niños de Buenos Aires. 2013;55:250.

13. Tigre A, Alvarado Cañizares J, Gerardo D, Rojas A, Andrés J. Análisis Situacional de la Atención en la Implementación de Triaje en el Servicio de Emergencia Pediátrica del Hospital José Carrasco Arteaga Cuenca. Cuenca, Ecuador; 2013. min.58: Med 1699.

14. Vázquez Carranza I, Fuentes Cuevas M. Relación de Urgencias Reales y Sentidas en un Servicio de Urgencias Pediátricas. Archivos de Investigación Materno Infantil. 2011;III,(1):19-23.

15. Gómez MB. Manual de Procedimientos, Recepción Acogida y Clasificación (RAC). PAVLICICH SONIA VIVIANA, Editor. Ministerio De Salud; Paraguay; 2011. 